\title{
CÁNCER TEMPRANO DE LA VESÍCULA BILIAR. TRATAMIENTO Y SOBREVIDA A LARGO PLAZO*
}

\author{
Drs. Jaime Castillo K. ${ }^{1}$, Attila Csendes J. ${ }^{1}$, Juan Carlos Díaz J. ${ }^{1}$, \\ Alexandre Sauré M. ${ }^{1}$, Matías Guajardo B. ${ }^{1}$, Hans Lembach J. ${ }^{1}$ \\ 1 Departamento de Cirugía, Hospital Clínico Universidad de Chile. \\ Santiago, Chile.
}

\section{Survival of patients with early gallbladder cancer}

Background: Advanced gallbladder cancer has a high mortality. Therefore, this tumor should be diagnosed and treated in early stages. Aim: To analyze the survival rates of patients with an early gallbladder cancer. Material and Methods: Retrospective analysis of medical records of patients with early gallbladder cancer diagnosed until 2003. From that date, all patients with gastrointestinal cancer were registered in a prospective record. Patients with early gallbladder cancer defined as Tis (Carcinoma in situ), T1A (Tumor invades lamina propia) and T1B (Tumor invades muscular layer), were included in this analysis. Results: Eighty patients aged 31 to 87 years $(74 \%$ women) were analyzed. The diagnosis was made during surgery in two patients and during the pathological study in the rest. All 24 patients with Tis tumors were alive after 96 months of follow up. Only one of 16 patients with T1A tumors died of unknown causes. Among the 40 patients with T1B tumors, 24 were re-operated and survival was over $90 \%$. Conclusions: This group of patients with early gallbladder cancer had a five years survival over $90 \%$. In patients with muscle involvement does not seem necessary to extend the surgery.

Key words: Gallbladder cancer, survival, early stage.

\section{Resumen}

Introducción: Dada la elevada mortalidad del cáncer vesicular en etapas avanzadas, lo ideal es diagnosticarlo y tratarlo en una etapa temprana de la enfermedad. Objetivo: Analizar la sobrevida de pacientes con carcinoma temprano de la vesícula biliar y el impacto de una eventual re-operación. Material y Método: Se incluyen en el estudio 80 pacientes con cáncer temprano, siendo la mayoría (65 pacientes) parte de un estudio prospectivo desde mayo de 2004 hasta febrero de 2013. Fueron divididos en 3 grupos: a) carcinoma in situ T1s; b) carcinoma mucoso T1a; c) carcinoma muscular I1b. Seguimiento en el $100 \%$ de los casos. Resultados: El diagnóstico de carcinoma temprano se hizo en 2 pacientes durante la cirugía, en el resto fue un hallazgo del estudio histo-patológico. Los 24 pacientes con carcinoma in situ estaban vivos a los 96 meses de seguimiento. De los 16 pacientes con carcinoma mucoso, sólo $1(6 \%)$ falleció sin conocerse la causa. Entre los 40 pacientes con cáncer muscular, hubo 24 que no se reoperaron y 16 reoperados. Los resultados anatomo

*Recibido el 2 de enero de 2014 y aceptado para publicación el 14 de abril de 2014.

Los autores declaran no tener conflicto de interés.

Correspondencia: Dr. Jaime Castillo K.

castillo_koch@yahoo.com 
patológicos fueron similares y la sobrevida de ambos grupos fue superior al $90 \%$. Conclusión: En pacientes con carcinoma de la vesícula biliar in situ y hasta la mucosa, la colecistectomía logra sobrevida mayor del $95 \%$ a largo plazo. En pacientes con carcinoma muscular, ésta se acompaña de una sobrevida mayor a $90 \%$ a 5 años y no parece justificarse ampliar la cirugía.

Palabras clave: Cáncer temprano, vesícula biliar, cáncer mucoso, cáncer muscular.

\section{Introducción}

El carcinoma de la vesícula biliar constituye la segunda causa de muerte por tumores malignos en la mujer chilena ${ }^{1-3}$, manteniendo una tasa de 11,3 fallecidos por 100.000 habitantes. Esto equivale a más de 1.500 mujeres fallecidas en un año. Una de las medidas para bajar esta cifra es diagnosticar este cáncer en etapas más precoces. El cáncer temprano de la vesícula corresponde al carcinoma in situ (TiS), el que compromete la mucosa hasta la lámina propia (T1a) y al que compromete hasta la capa muscular (T1b).

El propósito del presente estudio fue evaluar las características clínicas y anátomo-patológicas de un grupo de pacientes con carcinoma temprano de la vesícula biliar, como también determinar la sobrevida a largo plazo, compararando nuestros resultados con los publicados por otros autores nacionales.

\section{Material y Método}

\section{Pacientes estudiados}

El grupo corresponde a un total de 80 pacientes con cáncer temprano de la vesícula biliar entre enero de 1998 y febrero de 2013. Hasta el año 2003 los análisis eran retrospectivos y desde la creación de la Unidad de Oncología Quirúrgica en nuestro Departamento el año $2004^{4}$, todos los pacientes oncológicos se ingresan en forma prospectiva en una plantilla Excel con todos los datos completos y son sujetos a un estricto control posterior por la enfermera universitaria a cargo. Esto permite conocer con mucho detalle la evolución alejada y la sobrevida. Se excluyeron pacientes con carcinoma hasta la subserosa (T2) o etapas posteriores.

\section{Estudio anátomo-patológico}

Cada vesícula extirpada es procesada en su totalidad en cortes seriados y mapeados adecuadamente una vez pesquisado el carcinoma por el Departamento de Anatomía Patológica del hospital.

\section{Seguimiento}

Cada paciente es ubicado y controlado una vez por año por la Unidad de Oncología. Además se dispone de los certificados de defunción conociendo de esta manera la fecha exacta del fallecimiento.

\section{Análisis estadístico}

Para la prueba de significación estadística, se empleó el Test del $\chi^{2}$, tomando un $\mathrm{p}<0,05$ como significación estadística. La sobrevida actuarial se calcula según el método de Kaplan-Meier.

\section{Definiciones}

Se empleó la clasificación propuesta por la $6^{\text {th }}$ American Joint Commission on Cancer del año $2003^{5}$, que divide al carcinoma temprano en 3 subgrupos:

a.- T1S = carcinoma in situ.

b.- T1a = tumor invade mucosa hasta la lámina propia.

c.- $\mathrm{T} 1 \mathrm{~b}=$ tumor invade la capa muscular.

Esto comprende al estadio 0: TiS, N0, M0 o al estadio IA: T1, N0, M0.

\section{Resultados}

Hubo un total de 80 pacientes con carcinoma temprano de la vesícula biliar, correspondiente a 59 mujeres $(74 \%)$ y 21 hombres, con una edad promedio de 56 años. No hubo diferencias significativas entre los 3 subgrupos analizados (Tabla 1).

Entre el 8 y $12 \%$ de los pacientes eran asintomáticos, siendo el cólico biliar el síntoma que motivó la operación en un $90 \%$ mientras que ictericia se observó en cerca de 8 a $10 \%$ por coledocolitiasis asociada (Tabla 2). Colecistolitiasis estaba presente en el $100 \%$ de los casos.

En ningún paciente se sospechó el diagnóstico en el preoperatorio. Durante la operación se planteó

Tabla 1. Cáncer temprano de vesícula biliar. $\mathbf{N}=80$

\begin{tabular}{|lcccc|}
\hline & TiS & T1A & T1B & p \\
& $\mathbf{n = 2 4}$ & $\mathbf{n = 1 6}$ & $\mathbf{n = 4 0}$ & \\
Edad x & & & & \\
$\quad$ Años & 53,7 & 58,6 & 58,4 & n.s. \\
$\quad$ Rango & $35-81$ & $31-87$ & $31-80$ & \\
Género & & & & \\
$\quad$ F & $19(79 \%)$ & $11(73 \%)$ & $29(73 \%)$ & n.s. \\
M & 5 & 5 & 11 & \\
Asintomáticos & $8 \%$ & $12 \%$ & $12 \%$ & n.s. \\
Colecistolitiasis & $100 \%$ & $100 \%$ & $100 \%$ & \\
\hline
\end{tabular}


Tabla 2. Síntomas cáncer temprano de vesícula biliar. $\mathbf{N}=\mathbf{8 0}$

\begin{tabular}{|lcccl} 
& TiS & T1A & T1B & p \\
& $\mathbf{n = 2 4}$ & $\mathbf{n = 1 6}$ & $\mathbf{N = 4 0}$ & \\
Cólico & $92 \%$ & $88 \%$ & $93 \%$ & NS \\
Ictericia & $8 \%$ & $12 \%$ & $7 \%$ & NS \\
Sintomáticos & $92 \%$ & $88 \%$ & $88 \%$ & NS \\
\hline
\end{tabular}

Tabla 4. Cáncer temprano vesícula biliar. $\mathbf{N}=80$

\begin{tabular}{|lccc|}
\hline Evolución postoperatoria & $\begin{array}{c}\text { TiS } \\
\mathbf{n = 2 4}\end{array}$ & $\begin{array}{c}\text { T1a } \\
\mathbf{n}=\mathbf{1 6}\end{array}$ & $\begin{array}{c}\text { T1b } \\
\mathbf{n}=\mathbf{4 0}\end{array}$ \\
Morbilidad & 0 & $2(12 \%)$ & $2(5 \%)$ \\
$\quad$ Hemoperitoneo & & 1 & \\
$\quad$ Fístula biliar & & 1 & \\
$\quad$ Infección sitio qx. & & & 1 \\
$\quad$ Absceso subfrénico & & & 1 \\
Mortalidad & 0 & 0 & 0 \\
\hline
\end{tabular}

la posibilidad de un carcinoma en sólo 2 pacientes $(2,5 \%)$ que se convirtieron a cirugía laparotómica. En el resto el diagnóstico preciso se realizó con el estudio anátomo-patológico de la pieza extirpada.

La operación primaria (Tabla 3) fue una colecistectomía laparoscópica en 59 pacientes $(74 \%)$, mientras que en 21 pacientes se convirtió a un abordaje laparotómico o se realizó de inicio por vía laparotómica, ya sea por presencia de coledocolitiasis, fístula colecisto duodenal, esófago de Barrett, acalasia, etc. Reoperación para completar una cirugía oncológica se realizó con 19 pacientes, en especial en el 40\% de los casos con un T1b, usualmente 1 a 2 meses posterior a la cirugía inicial. La evolución postoperatoria fue sin incidentes en $95 \%$ y no hubo mortalidad postoperatoria. Las complicaciones postoperatorias fueron 1 hemoperitoneo, 1 fístula biliar externa y 2 infecciones localizadas (Tabla 4).

En la Tabla 5 se muestra el resultado de los hallazgos clínicos y anátomo-patológicos en los 3 subgrupos de pacientes. No se evidenció infiltración del hígado o lecho de la vesícula en ninguno.

Sólo en 1 paciente se comprobó una infiltración del conducto cístico.

El análisis histológico de los linfonodos resecados se aprecia en la Tabla 6. Hubo sólo un linfonodo con metástasis en el grupo de carcinoma mucoso.

El seguimiento de los pacientes (Tabla 7) fue de $100 \%$ con un promedio de 96 meses (12-219). Los 24 pacientes con carcinoma in situ estaban vivos
Tabla 3. Cáncer temprano de vesícula biliar

\begin{tabular}{|lccc|}
\hline Operaciones & $\begin{array}{c}\text { TiS } \\
\mathbf{n}=\mathbf{2 4}\end{array}$ & $\begin{array}{c}\text { T1a } \\
\mathbf{n = 1 6}\end{array}$ & $\begin{array}{c}\text { T1b } \\
\mathbf{n}=\mathbf{4 0}\end{array}$ \\
Operación inicial & & & \\
Laparoscópica & 19 & 11 & 29 \\
Laparotómica & 5 & 5 & 11 \\
Reoperación & 0 & $3(19 \%)$ & $16(40 \%)$ \\
Tiempo (meses) & $1,2(1-2)$ & $1,4(1-2)$ & \\
\hline
\end{tabular}

Tabla 5. Cáncer temprano de vesícula biliar

\begin{tabular}{|lccc|}
\hline Análisis histológico & $\begin{array}{c}\text { TiS } \\
\mathbf{n = 2 4}\end{array}$ & $\begin{array}{c}\text { T1a } \\
\mathbf{n = 1 6}\end{array}$ & $\begin{array}{c}\text { T1b } \\
\mathbf{n = 4 0}\end{array}$ \\
$\begin{array}{l}\text { Resección hepática } \\
\text { IVb }+ \text { V }\end{array}$ & 0 & 3 & $\begin{array}{c}16 \\
(19 \%)\end{array}$ \\
$\begin{array}{l}\text { Infiltración lecho } \\
\text { vesicular (+) }\end{array}$ & & 0 & 0 \\
Infiltración vía biliar & 0 & 0 & $\begin{array}{c}1(2 \%) \\
\text { conducto cístico }\end{array}$ \\
\hline
\end{tabular}

Tabla 6. Cáncer temprano de vesícula biliar

\begin{tabular}{|lccc|}
\hline Análisis linfonodos & TiS & T1a & T1b \\
\hline n linfonodos resecados & 5 & 48 & 137 \\
n linfonodos positivos & 0 & $1(2 \%)$ & 0 \\
\hline
\end{tabular}

Tabla 7. Cáncer temprano de vesícula biliar

\begin{tabular}{|lccc|}
\hline Seguimiento & $\begin{array}{c}\text { TiS } \\
\mathbf{n}=\mathbf{2 4}\end{array}$ & $\begin{array}{c}\text { T1a } \\
\mathbf{n}=\mathbf{1 6}\end{array}$ & $\begin{array}{c}\text { T1b } \\
\mathbf{n}=\mathbf{4 0}\end{array}$ \\
Seguimiento & $100 \%$ & $100 \%$ & $100 \%$ \\
Control alejado meses & 87 & 105 & 98 \\
Rango & $(14-152)$ & $(13-175)$ & $(12-219)$ \\
\% sobre 60 meses & 88 & 69 & 70 \\
\hline
\end{tabular}

Tabla 8. Cáncer temprano de vesícula biliar

\begin{tabular}{|lccl|}
\hline Sobrevida & $\begin{array}{c}\text { TiS } \\
\mathbf{n}=\mathbf{2 4}\end{array}$ & $\begin{array}{c}\text { T1a } \\
\mathbf{n}=\mathbf{1 6}\end{array}$ & $\begin{array}{l}\text { T1b } \\
\mathbf{n}=\mathbf{4 0}\end{array}$ \\
Vivos & $24(100 \%)$ & $15(94 \%)$ & $37(92,5 \%)$ \\
Fallecidos & 0 & $1(6 \%)$ & $\begin{array}{l}3(7,5 \%) \\
\text { - Carcinomatosis peritoneal } \\
\end{array}$ \\
& & & - Metástasis hepáticas \\
\hline
\end{tabular}


Tabla 9. Cáncer temprano de vesícula biliar

\begin{tabular}{|c|c|c|}
\hline $\begin{array}{l}\text { Etapa T } 1 b \\
n=40\end{array}$ & $\begin{array}{c}\text { No operados } \\
n=24\end{array}$ & $\begin{array}{c}\text { Re-operados } \\
n=16\end{array}$ \\
\hline $\begin{array}{l}\text { n linfonodos } \\
\text { resecados/positivos }\end{array}$ & $28 / 0$ & $109 / 0$ \\
\hline Infiltración vía biliar & 1 (cístico) & 0 \\
\hline $\begin{array}{l}\text { Infiltración lecho } \\
\text { hepático }\end{array}$ & 0 & 0 \\
\hline Sobrevida global & $22(92 \%)$ & $15(94 \%)$ \\
\hline Fallecidos & $\begin{array}{c}2 \\
(1 \text { desconocido }) \\
1 \text { carcinomatosis peritoneal }\end{array}$ & $\begin{array}{c}1 \\
\text { (metástasis } \\
\text { peritoneal) }\end{array}$ \\
\hline Seguimiento (meses) & 98 & 92 \\
\hline
\end{tabular}

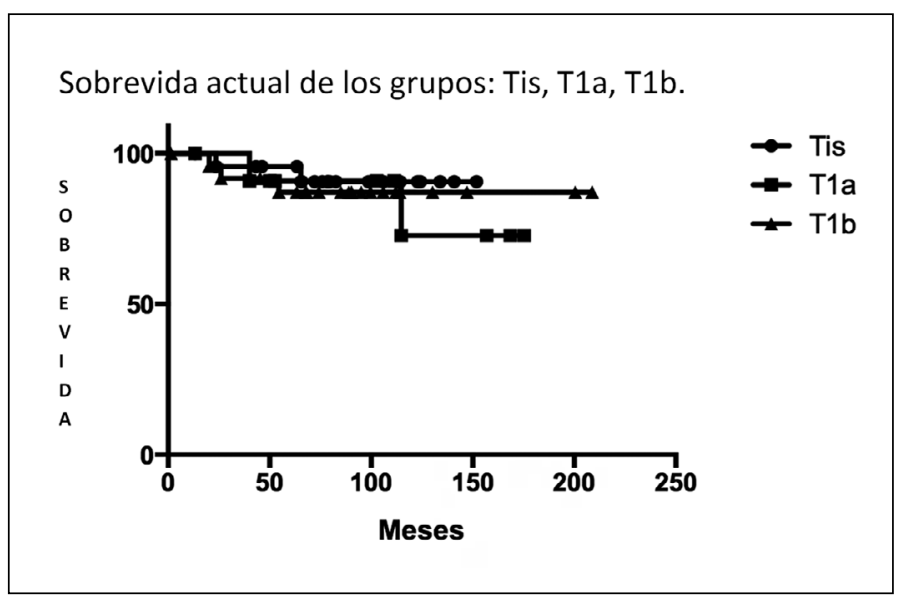

Figura 1. Sobrevida a largo plazo de pacientes con cáncer temprano de la vesícula biliar.

\section{Curva sobrevida actual de T1b operados y no operados}

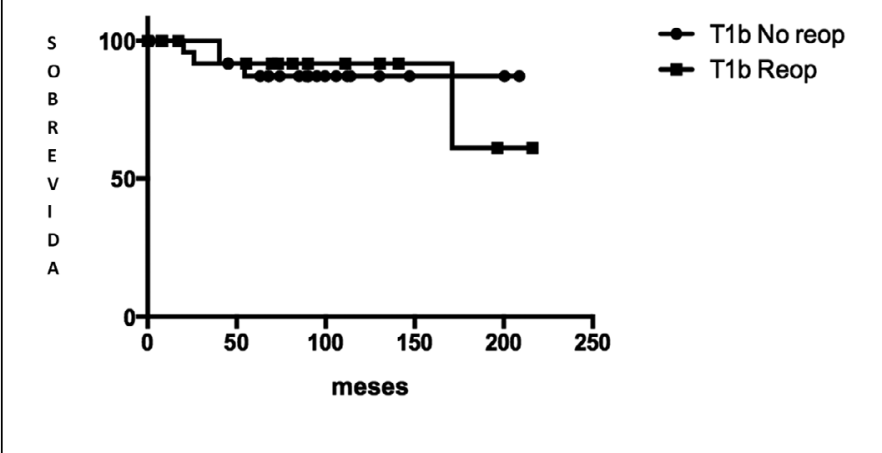

Figura 2. Sobrevida a largo plazo de pacientes con carcinoma hasta la capa muscular de la vesícula biliar. al control alejado (Tabla 8). Sólo uno (6\%) de los casos con cáncer mucoso falleció a los 40 meses de operado, sin conocerse claramente la causa. Entre los 40 pacientes con cáncer muscular, 3 fallecieron (7,5\%); uno a los 20 meses por carcinomatosis peritoneal, uno a los 40 meses por metástasis hepática y uno a los 26 meses por causa desconocida.

Al separar los 40 pacientes con cáncer muscular en re-operados o no, en la Tabla 9 se aprecia que hubo 24 que no se reoperaron y 16 re-operados. Los resultados de los hallazgos anátomopatológicos fueron similares y la sobrevida en ambos grupos fue superior al $90 \%$ con un seguimiento promedio de 95 meses.

La Figura 1 muestra la sobrevida muy alejada de los 3 grupos. Los resultados del seguimiento hasta 200 meses muestran sobrevida muy similar entre Tis y T1a, mientras que pacientes con T1b tienen una menor sobrevida pero no estadísticamente diferente $(\mathrm{p}>0,7)$. La Figura 2 señala la sobrevida comparada con pacientes $\mathrm{T} 1 \mathrm{~b}$ re-operados o no. Los resultados alejados son enteramente similares, sin diferencias significativas $(p>0,9)$.

\section{Discusión}

Los resultados del presente estudio sugieren en primer lugar que el cáncer de la vesícula biliar diagnosticada en una etapa temprana, tiene por lo menos $90 \%$ de sobrevida a 5 años. En segundo lugar, los resultados obtenidos no justifican una re-operación o re-cirugía en pacientes con cáncer hasta la capa muscular (T1b), pese a tener una menor sobrevida no estadísticamente significativa.

El carcinoma de la vesícula biliar es un tumor muy frecuente en las mujeres chilenas con una tasa de mortalidad cercana a $11,3 \times 100.000$ mujeres $^{2,3,6}$. Al realizar colecistectomía rutinaria en pacientes con colecistolitiasis, la incidencia global de carcinoma vesicular es cercana al $3 \%^{7-11}$. Este hallazgo incidental de carcinoma de la vesícula es muy importante, ya que en este grupo el cáncer temprano ocurre entre 
el 20 a $30 \%$ de los $\operatorname{casos}^{11-14}$, contrastando con la incidencia de cáncer temprano en pacientes que llegan a la cirugía con el diagnóstico preoperatorio de carcinoma, en cuyo caso la incidencia con un tumor temprano es menos del 5\% $\%^{8,9,15-18}$. La importancia de este hallazgo radica en que en la literatura nacional se describe una sobrevida a 5 años de hasta $90 \%$ en estos pacientes ${ }^{9,12-15,18-20}$. Nuestros resultados confirman estos reportes previos y corresponden a la mayor casuística chilena después de los trabajos publicados por De Aretxabala y Roa ${ }^{12,19-21}$.

El cuadro clínico es enteramente similar a los pacientes con colecistolitiasis, siendo asintomáticos sólo el $10 \%$, por lo que es imposible diagnosticar este cáncer antes de la intervención quirúrgica. De hecho, sólo en el 2,5\% de nuestros pacientes se sospechó la presencia de un carcinoma, cifra similar al $5 \%$ reportado por De Aretxabala ${ }^{21}$.

Una vez completado el estudio anatomopatológico, el carcinoma temprano se divide en 3 grupos: carcinoma in situ, carcinoma de la capa mucosa y carcinoma de la capa muscular. La vesícula biliar no tiene submucosa. En los 2 primeros grupos no hay ninguna duda que la cirugía fue completamente curativa sólo con la colecistectomía laparoscópica y se obtiene una sobrevida a 5 años mayor a $95 \%$, por lo que no requieren de otra intervención oncológica o quirúrgica. Por el contrario, pacientes en etapa $\mathrm{T} 1 \mathrm{~b}$ han generado controversia. En la literatura internacional se ha descrito hasta $16 \%$ de compromiso linfático y sobrevida cercana de $70 \%{ }^{22,23}$, pero la mayoría de los autores concluyen que la simple colecistectomía es suficiente como terapia definitiva en estos $\operatorname{casos}^{24-27}$. En Chile, De Aretxabala y Roa han llegado a conclusiones similare ${ }^{21,28}$ a lo reportado también por nuestro grupo ${ }^{2,18}$. Estos resultados llegaron a formular un consenso nacional respecto al manejo del carcinoma de la vesícula biliar ${ }^{1}$. Los resultados obtenidos en el presente estudio confirman esta política.

En el presente estudio no se apreció ninguna ventaja sustantiva al realizar una re-operación que incluía segmentectomía hepática IVb y $\mathrm{V}$ más disección linfonodal en pacientes etapa $1 b$, ya que la sobrevida fue enteramente similar. La indicación de la re-intervención en pacientes con un cáncer muscular en la biopsia diferida fue por dos motivos; el primero, debido a que en la biopsia de la colecistectomía laparoscópica no se informaba el ganglio cístico y por ende se desconocía el estadio linfonodal para la adecuada clasificación; el segundo motivo, fue la aceptación y la solicitud del paciente a ser re-intervenido una vez explicado extensamente los beneficios y riesgos de la colecistectomía extendida (hepatectomía segmentaria IVb y V, con disección linfonodal).
La discusión de si la colecistectomía preventiva disminuye la incidencia de cáncer vesicular está aún en evaluación. Hay evidencias que así lo sugieren ${ }^{2,16}$. En nuestro país aún no se ha evaluado el impacto de la política del AUGE en la colecistectomía preventiva de pacientes entre 35 y 49 años. Un estudio reciente ${ }^{11}$ del grupo de Valdivia no encontró un mayor impacto de esta política y sugería prolongar la edad hasta 55 años. Falta evaluar científicamente este tópico.

En conclusión, el carcinoma temprano de la vesícula biliar se diagnostica en la inmensa mayoría después de la colecistectomía. La sobrevida en estos pacientes es altísima, sobre $90 \%$ a 5 años. No se justifica una re-operación en pacientes etapa $\mathrm{T} 1 \mathrm{~b}$, es decir, con infiltración tumoral hasta la capa muscular.

\section{Referencias}

1. De Aretxabala X. Cáncer vesícula biliar: consideraciones luego del consenso. Rev Chil Cir. 2007;59:472-5.

2. De Aretxabala X, Maluenda F. Cáncer de vesícula biliar. Rev. Hosp Clínico U. Chile 2005;16:5-13.

3. Andia M, Gederteni A, Fureccio C. Cáncer de vesícula biliar: tendencias y distribución del riesgo en Chile. Rev Med Chile. 2006;134:565-74.

4. Cortés S, Csendes A, Yarmuch J. Resultados de la unidad de oncología del Departamento de Cirugía de un hospital universitario (2004-2010). Rev Chil Cir. 2011;5:534-7.

5. AJCC Cancer Staging Manual: American Joint Commission on Gallbladder Cancer, 6th Editor Philadelphia, Lippincott Ravers, 2003.

6. Candia M, Genderlivi A, Fureccio C. Cáncer vesícula biliar. Tendencias y distribución del riesgo en Chile. Rev Med Chile 2006;134:565-76.

7. Csendes A, Becerra M, Smok G, Medina E, Maluenda F. Prevalencia de cáncer de vesícula biliar en colecistectomías. Rev Med Chile 1991;119:887-90.

8. Guzmán P, Pávez J, Consini N, Navarro S, Handorf L. Patología de la vesícula biliar en la $\mathrm{V}$ región. Rev Chil Cir. 2000;52:465-70.

9. Venturelli F, Cárcamo C, Born M, Venegas L, Beltrán M, Venturelli A, y cols. Cáncer vesícula en colecistectomías. Análisis sobrevida y factores pronósticos. Rev Chil Cir. 2008;60:271-80.

10. Ventureli F, Cárcamo C, Born M, Venegas L, Bertrán V. Cáncer vesicular en colecistectomías: análisis de sobrevida y factores pronósticos. Rev Chil Cir. 2008;60:27781.

11. Gómez B, Celedón E, Venturelli A, Díaz J, Barese C, Cárcamo C. Colecistectomía preventiva del cáncer vesicular en adultos de 35 a 49 años. Cuad Cir. 2009;33:1520.

12. Roa I, De Aretxabala X, Araya JC, Villaseca M, Roa JC, Ibacache G. Elementos morfológicos pronósti- 
cos en el cáncer de la vesícula biliar. Rev Med Chile 2002;130:387-95.

13. Cortés L, Angel B, Castro M, Adelsdofer-Carter J. Resultados en cirugía del cáncer de la vesícula biliar. Bol. Hosp. Viña del Mar 2005;61:12-6.

14. Talbot C, Klaasen R, Huscientes R, Díaz M, Lima R. Sobrevida en cáncer de vesícula biliar estadio I. Rev Chil Cir. 2003;55:65-9.

15. Galielli M, Hugo S, Domínguez A, Baez S, Venturelli A, Puga M, y cols. Letalidad del cáncer de vesícula biliar es independiente del lugar de atención o características demográficas. Rev Med Chile 2010:138:1357-61.

16. Maluenda F, Díaz JC, De Aretxabala X, Burdiles P, Csendes A, Contreras L. Aspectos quirúrgicos del cáncer de la vesícula biliar. Rev Med Chile 2005;133:72328.

17. Muñoz C, Davanzo C, Cárdenas B, Naranjo B, Luengas $\mathrm{R}$, Lanzarini E, y cols. Prevalencia de displasia en vesícula biliar. Rev Chil Cir. 2008;60:198-01.

18. Maluenda F. Cáncer de la vesícula biliar. Resultados de la cirugía resectiva. Rev Chil Cir. 2002;54:464-73.

19. Roa I, Muñoz S, Ibacache G, De Aretxabala X. Desde la displasia hasta el cáncer de la vesícula biliar. Rev Med Chile 2009;137:873-80.

20. Roa I, De Aretxabala X, Roa JC, Araya J, Villaseca M, Guzmán P, y cols. ¿Es el cáncer de la vesícula biliar una enfermedad de mal pronóstico en Chile? Rev Med Chile 2002; 130:1295-02.

21. De Aretxabala X, Roa I, Mora J, Pincheira O, Burgos L,
Silva J, y cols. Cáncer de la vesícula biliar: manejo de pacientes con invasión de la técnica muscular. Rev Med Chile 2004;132:183-88.

22. Ogura Y, Mizumoto R, Isaji S, Kusuda T, Matsuda S, Tabata M. Radical operations for carcinoma of the gallbladder. Present status in Japan. World J Surg. 1991;15:337-43.

23. Wagholikar GD, Behari A, Krishnani N, Kumar A, Sikora SS, Saxena R, et al. Early gallbladder cancer. J Am Coll Surg. 2002;194:137-41.

24. You DD, Lee HG, Paik KY, Heo JS, Choi SH, Choi DW. What is an adequate extent of resection for T1 gallbladder cancers? Ann Surg. 2008;247:835-38.

25. Wakai T, Shirai N, Yokoyama N. Early gallbladder carcinoma does not warrant radical resection. Br J Surg. 2001;88:675-78.

26. Shirai Y, Yoshida K, Tsukada K, Muto T. Inapparent carcinoma of the gallbladder. An appraisal of a radical second operation after simple cholecystectomy. Ann Surg. 1992;215:326-31.

27. Otero JC, Proske A, Vallilengua C, Luján M, Poletto L, Pezzotto SM, et al. Gallbladder cancer: surgical results after cholecystectomy in 25 patients with lamina propia invasion and 26 patients with muscular layer invasion. J. Hepatobiliary Pancreat Surg. 2006;13:56266.

28. De Aretxabala X, Roa I, Hepp J, Maluenda F, Mordojovich $\mathrm{G}$, Leon $\mathrm{J}$, et al. Early gallbladder cancer: is further treatment necessary. J Surg Oncol. 2009;100:589-93. 